\section{Coming Events}

Due to COVID-19, please check to see if the listed events have been postponed or cancelled.

2020

16th International Congress of Histochemistry and Cytochemistry (ICHC)

August 30-September 2, 2020

Prague, Czech Republic

http://ichc2020.com

Neuroscience 2020

October 24-28, 2020

Washington, DC

www.sfn.org/meetings/neuroscience-2020

\section{MRS Fall Meeting \& Exhibit}

November 29-December 4, 2020

Boston, MA

www.mrs.org/fall2020

\section{ASCB 2020 Annual Meeting}

December 5-9, 2020

Philadelphia, PA

www.ascb.org/meetings-events/future-ascb-meetings

2021

Microscopy \& Microanalysis 2021

August 1-5, 2021

Pittsburgh, PA

www.microscopy.org

2022

Microscopy \& Microanalysis 2022

July 31-August 4, 2022

Portland, OR

www.microscopy.org

\section{3}

Microscopy \& Microanalysis 2023

July 24-28, 2023

Minneapolis, MN

www.microscopy.org

\section{4}

Microscopy \& Microanalysis 2024

July 28-August 1, 2024

Cleveland, $\mathrm{OH}$

www.microscopy.org

\title{
Opening a New Window for Observing Embryogenesis
}

\section{Stephen W. Carmichael}

Mayo Clinic, Rochester, MN 55905

carmichael.stephen@mayo.edu

Embryonic development is a complex process that is difficult to observe directly. Histologic examination of fixed embryos does not capture the dynamics of development. Other imaging methods have limitations, such as low resolution and the inability to take advantage of transgenic strains with fluorescent reporters. Studies with superresolution microscopy of specimens in vitro has limitations, such as the inability to mimic the uterine environment past embryonic day 9 (E9) when various organs form.

A recent study [1] by a large international group led by Qiang Huang, Rudolf Jaenisch, and Xiling Shen demonstrated that it is possible to overcome some of these limitations by implanting a window into the uterus of pregnant mice (dams). This allows intravital imaging that can be used to observe the formation of organs at high resolution from E9.5 to birth. The embryo becomes easily identifiable under a dissection microscope at about E9.5 when the allantois fuses with the chorionic plate resulting in a large surface area for gas and nutrient exchange.

Initially an optical window (and a clip to stabilize the window) was designed by Huang et al. and fabricated by $3 \mathrm{D}$ printing. A round incision was made in the abdominal wall, and some skin was removed. The uterus was exteriorized, and an embryo near an ovary was selected. The window was implanted in the uterine wall, the embryo was sutured to the abdominal muscle, the abdominal muscle was sutured to

\section{Fluorescein diffusion into the embryo}
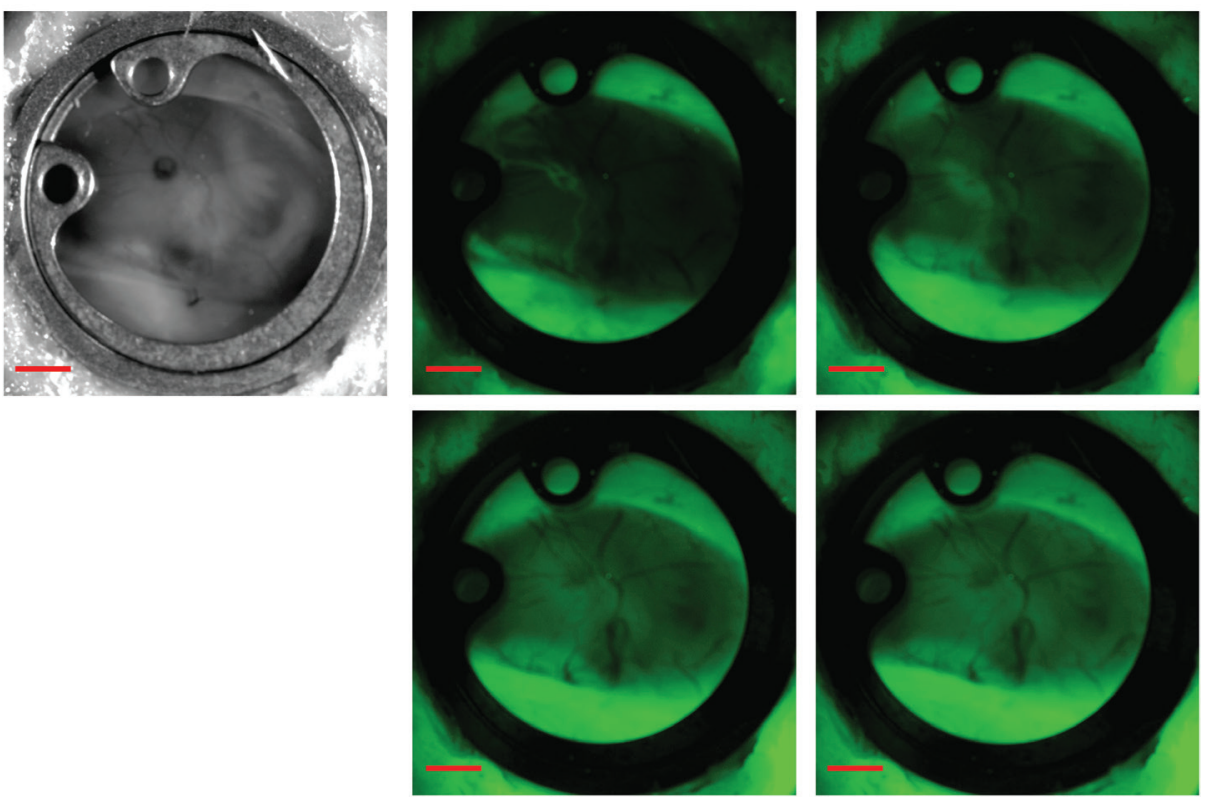

Figure 1: The image in the upper left corner is of an embryo at E15.5. The color images show diffusion of fluorescein into an embryo after it was injected into the dam. The upper left color image was taken 1.5 minutes after the injection, the upper right image taken at 15 minutes, lower left image taken at 30 minutes, and lower right at 15 minutes. Scale bar $=2 \mathrm{~mm}$. 
First $\gg \gg\rangle$

\section{HIGH THROUGHPUT SEM System}

\section{Navigator-ITE}

(6) 10 times faster than

conventional FE SEMs

(1). Direct electron detection technology and Dual channel imaging (SE\&BSE)

(I) Intelligent atlas and cross-scale material characterization

(远) Big data intelligent analysis and $\mathrm{Al}$ computing
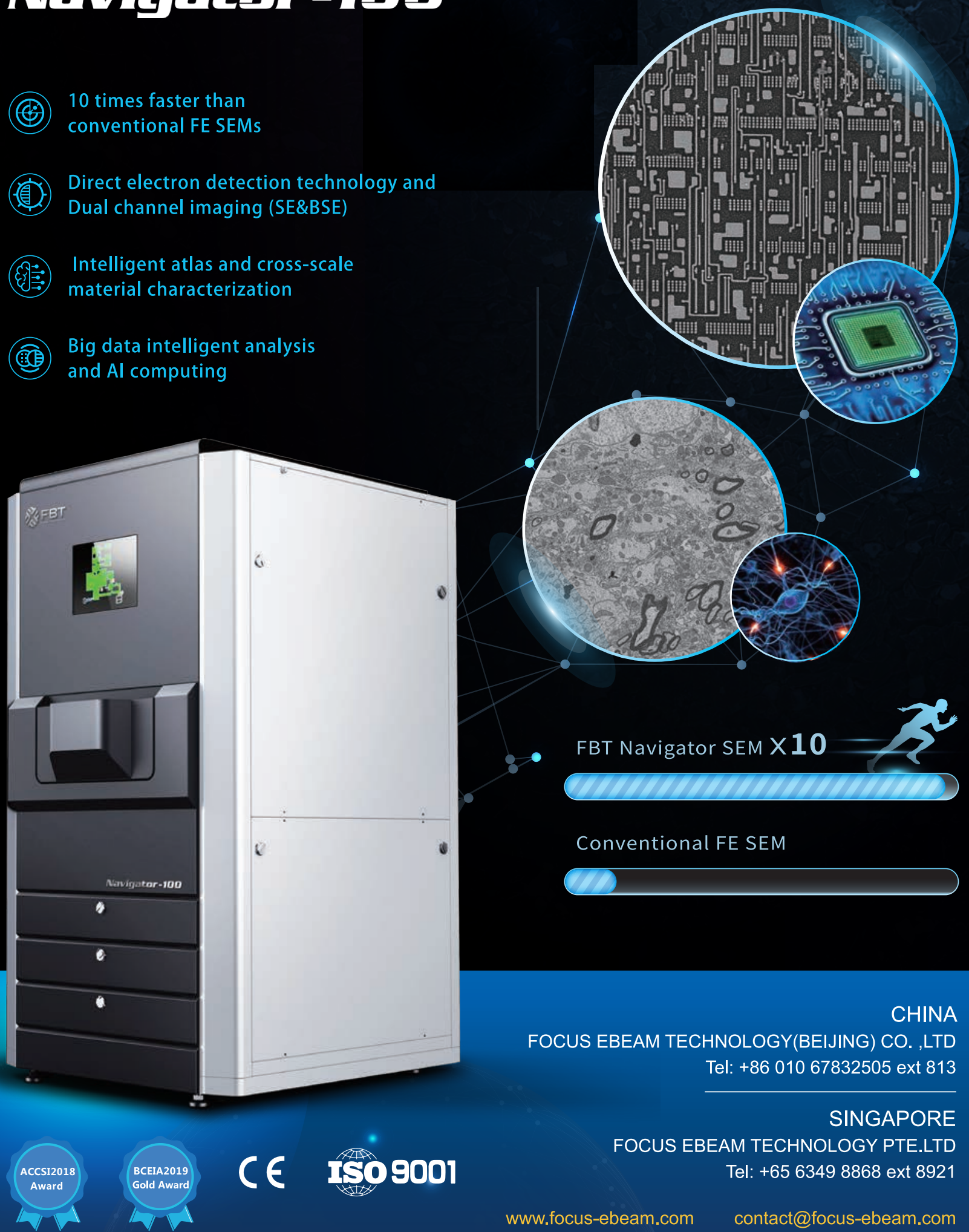

CHINA

FOCUS EBEAM TECHNOLOGY(BEIJING) CO. ,LTD

Tel: +86 01067832505 ext 813

SINGAPORE

FOCUS EBEAM TECHNOLOGY PTE.LTD

Tel: +65 63498868 ext 8921 
the window, and the window was secured in position with glue. This minimized motion from the dam's breathing. The initial window was circular with a $10 \mathrm{~mm}$ inner diameter and $1.5 \mathrm{~mm}$ depth. It was replaced with a larger, elliptical window (long axis $15 \mathrm{~mm}$ ) during the later stages. The window was covered with a glass cover slip that could be removed for manipulating the embryo.

For the early stages the decidua and uterine muscle were stripped to improve visualization of the embryo. After E12.5 the decidua diminished, and the uterine wall became transparent, so there was no need for the stripping procedure. These procedures did not interfere with embryo survival in most cases as indicated by a beating heart. Later histological examination using hematoxylin and eosin staining showed no structural abnormalities in the embryos underneath the window. After the pups were born they suckled normally, and pups imaged as embryos under the window were indistinguishable from their littermates. They grew normally without noticeable abnormalities.

Transgenic mice with cell lineage-specific expression of fluorescent reporter are commonly used to study development in live organisms. Huang et al. used stereoscopic and two-photon microscopy to observe embryos in transgenic (for example, Wnt1-Cre-tdTomato) mice. They conducted a series of studies that not only provided proof of concept for the use of these windows, but also resulted in important information about embryogenesis. Briefly, the studies observed transient neurotransmission and early vasculatization neural crest cell (NCC)-derived perivascular cells in the brain, autophagy in the retina, viral gene delivery, and chemical diffusion through the placenta (Figure 1). They combined the imaging window with increasing the permeability of plasma membranes using an electrical field (electroporation) to label and track cell division and movement within embryos in utero. In addition, they observed that clusters of mouse NCC-derived cells expanded in interspecies chimeras, whereas adjacent human donor NCC-derived cells shrank.

Huang et al. have developed an intravital imaging window for visualizing and manipulating live mouse embryos in vivo from E9.5 until birth (around E20). This elegant study demonstrates the capability to image whole embryos in utero at high resolution, opening many new avenues for investigation: brain formation, peripheral nerve development, placental development, birth defects, gene editing, development of the immune system, environmental effects, and interspecies chimeras. It will be fascinating to see what new discoveries in embryogenesis are revealed using this exciting new technique!

\section{References}

[1] Huang et al., Science 368 (2020) 181-86.

[2] The author gratefully acknowledges Drs. Qiang Huang, Rudolf Jaenisch, and Xiling Shen for reviewing this article.

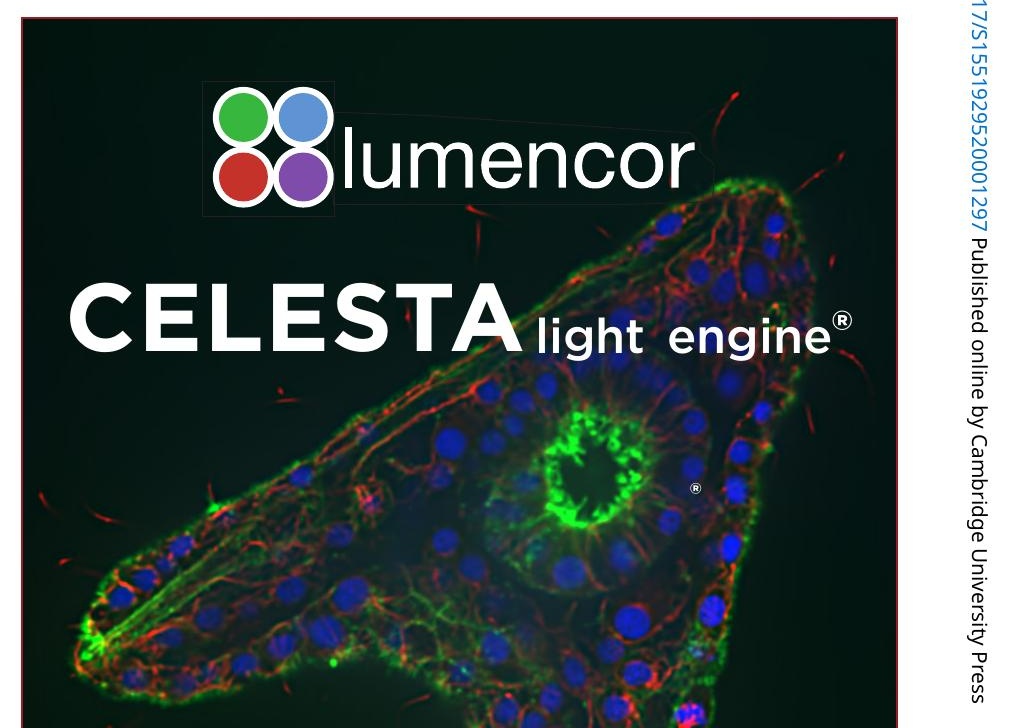

THE NEXT GENERATION LASERS COME TO LAHT

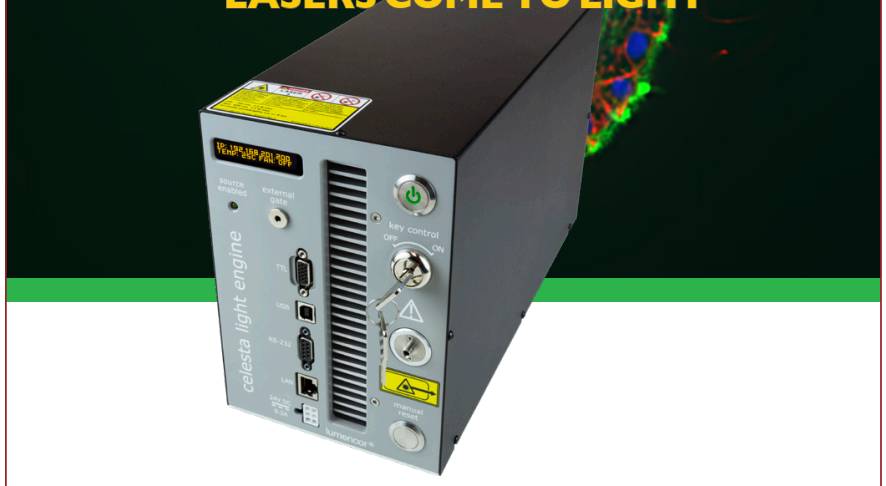

- Spectral breadth: 405, 446, 477, 520, $546,638,749 \mathrm{~nm}$

- Brightness: Seven lasers $1.1 \mathrm{~W} / \mathrm{mm}^{2} \mathrm{sr}$ at the distal end of a $1.5 \mathrm{~mm}$ diameter fiber

- Power: $1000 \mathrm{~mW} /$ line at distal end of 1.5 $\mathrm{mm}$ diameter fiber

- Control: Active power stabilization

- Stability: Exceptional reproducibility, ideal for quantitation

- Ease of use: Pre-aligned lasers

- Applications: Confocal, MERFISH, superresolution microscopy, photoactivation, optogenetics, FRAP, DNA-PAINT +++

- Customization: Off-the-shelf and OEM configurations upon request 


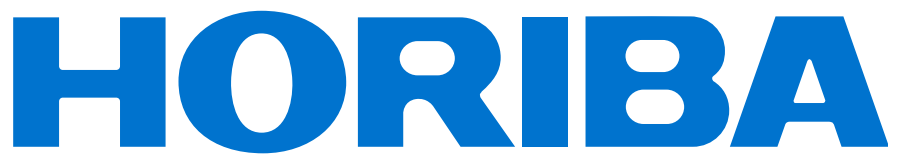

Scientific

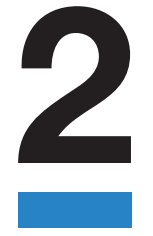

NEW

Innovative

Microscopy

Products

from HORIBA

Applications like

FLIM, TRPL,

photoluminescence,

Raman and

electroluminescence

require more

capabilities than your microscope can provide.

HORIBA has two new

products to help you

multitask your microscope.

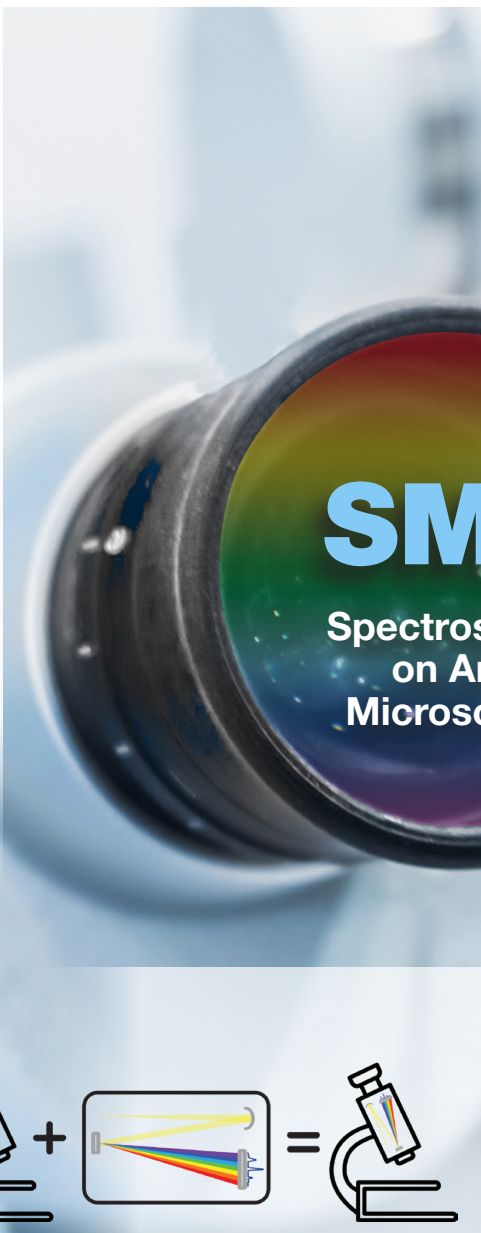

\section{NEW Standard Microscope Systems}

Add various spectroscopies to

ANY STANDARD Microscope

without sacrificing performance of either one!

\section{NEW SPAD FLIMera Camera}

Fluorescence lifetime images from

25K simultaneous pixel measurements

at a video rate of 30 frames per second!

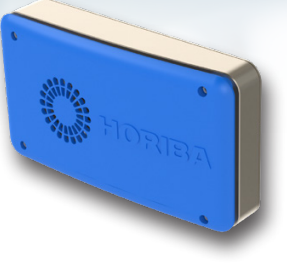

\begin{tabular}{|c|}
\hline ELEMENTAL ANALYSIS \\
\hline FLUORESGENGE \\
\hline BATINGS \& OEM SPEGTROMETERS \\
\hline OPTICAL GOMPONENTS \\
\hline FORENSIES \\
\hline PARTICLE GHARAGTERIZATION \\
\hline RAMAN / AFM-RAMAN / TERS \\
\hline SPEGTROSGOPIC ELLPSOMETRY \\
\hline SPR IMAGING \\
\hline $\begin{array}{c}\text { JOBIN YVON } \\
\text { Technology }\end{array}$ \\
\hline
\end{tabular}




\section{IIICIOSCONY \\ Innovation Awards}
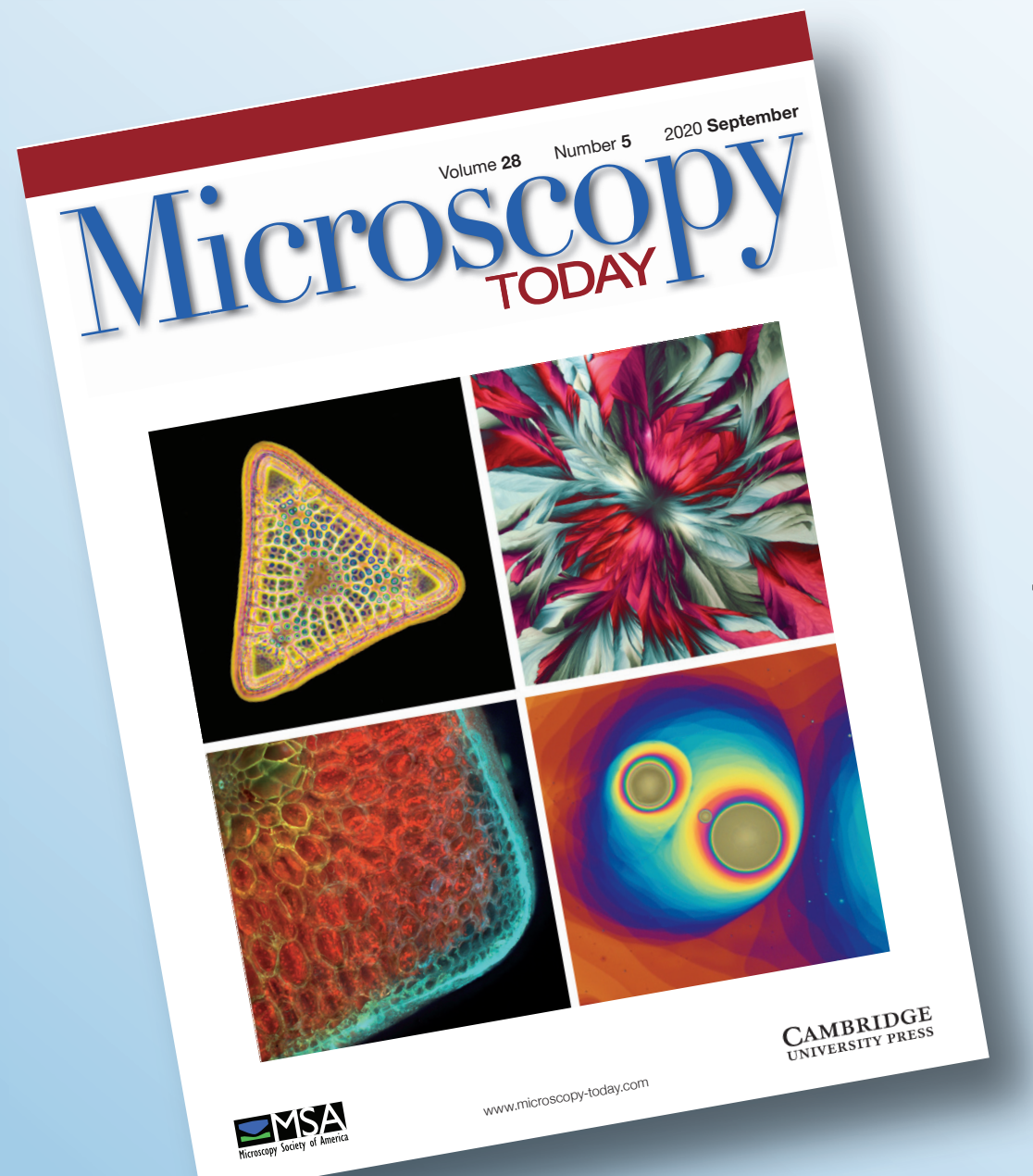

See this issue for details about the 2020 winners

Request application forms by email: charles.lyman@lehigh.edu Next deadline - March 22, 2021 


\section{Seeing the future with clarity}

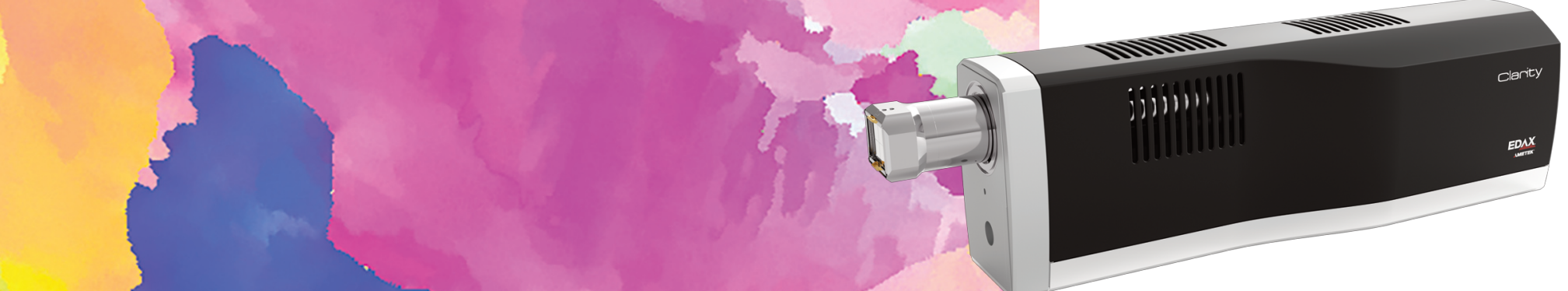

\section{Introducing the Clarity EBSD Analysis System}

Clarity $^{\mathrm{TM}}$ - the world's first Electron Backscatter Diffraction (EBSD) specific detector based on Direct Detection technology. This revolutionary approach provides unparalleled pattern quality, ultimate sensitivity, and distortion-free imaging, opening new doors to the evolution of EBSD pattern analysis.

- Direct electron detection of EBSD patterns

- Zero distortion for ultimate sharpness and maximum details

- No read noise for high sensitivity

- Single-electron detection

- True quantitative intensity measurements

- Ideal for beam-sensitive materials and HR-EBSD 